\title{
Collaborations vertueuses entre coopératives et municipalités
} Le cas historique de l'Émillie-Romagne après la Seconde Guerre mondiale

\author{
The virtuous partnership between cooperatives and local \\ government \\ The historic example of Emilia Romagna after the Second \\ World War
}

\section{Tito Menzani}

Numéro 321, juillet 2011

URI : https://id.erudit.org/iderudit/1020868ar

DOI : https://doi.org/10.7202/1020868ar

Aller au sommaire du numéro

\section{Éditeur(s)}

Association Recma

\section{ISSN}

1626-1682 (imprimé)

2261-2599 (numérique)

Découvrir la revue

Citer cet article

Menzani, T. (2011). Collaborations vertueuses entre coopératives et municipalités : le cas historique de l'Émilie-Romagne après la Seconde Guerre mondiale. Revue internationale de l'économie sociale, (321), 99-111. https://doi.org/10.7202/1020868ar

\section{Résumé de l'article}

Dans cet article, Tito Menzani analyse les synergies historiques entre l'action politique municipale et cette forme entrepreneuriale particulière qu'est la société coopérative en Émilie-Romagne, l'un des bastions mondiaux de la coopération, de la fin de la Seconde Guerre mondiale au début des années 70. Les relations entre le monde coopératif et les administrations publiques ont été particulièrement étroites, voire exclusives, entre 1943 et 1950 . Soutenue par les diverses forces politiques progressistes, et particulièrement par le Parti communiste en Émilie-Romagne, la coopération a joué un rôle de premier plan dans la reconstruction morale et matérielle du pays. Les coopératives ont contribué à la modernisation des campagnes et du commerce et à résorber la crise du logement. À partir des années 60, le mouvement coopératif n’est devenu qu'un des différents interlocuteurs des administrations publiques locales, avec lesquelles il a continué à partager une même volonté de justice sociale. L'auteur soutient la thèse selon laquelle cette collaboration a été un vecteur de moralisation de la vie publique dans les territoires, du fait de l'exigence éthique qui sous-tend la pratique coopérative 


\section{COLLABORATIONS VERTUEUSES ENTRE COOPÉRATIVES ET MUNICIPALITÉS}

Le cas historique de l'Emilie-Romagne après la Seconde Guerre mondiale de Bologne. Mél.: tito.menzani2@ unibo.it.

** Texte traduit de l'italien par Bernard Stauffer.
* Professeur auxiliaire, université

\author{
par Tito Menzani*
}

Dans cet article, Tito Menzani analyse les synergies historiques entre l'action politique municipale et cette forme entrepreneuriale particulière qu'est la société coopérative en Emilie-Romagne, l'un des bastions mondiaux de la coopération, de la fin de la Seconde Guerre mondiale au début des années 70. Les relations entre le monde coopératif et les administrations publiques ont été particulièrement étroites, voire exclusives, entre 1943 et 1950. Soutenue par les diverses forces politiques progressistes, et particulièrement par le Parti communiste en EmilieRomagne, la coopération a joué un rôle de premier plan dans la reconstruction morale et matérielle du pays. Les coopératives ont contribué à la modernisation des campagnes et du commerce et à résorber la crise $d u$ logement. A partir des années 60, le mouvement coopératifn'est devenu qu'un des différents interlocuteurs des administrations publiques locales, avec lesquelles il a continué à partager une même volonté de justice sociale. L'auteur soutient la thèse selon laquelle cette collaboration a été un vecteur de moralisation de la vie publique dans les territoires, du fait de l'exigence éthique qui sous-tend la pratique coopérative ${ }^{* *}$.

(1) Feltri, Brunetta (2007) et Capproti (2007) ont rajeuni une tradition conservatrice anticoopérative. V. Feltri est le directeur éditorial de Libero, quotidien populiste de droite. R. Brunetta est ministre du quatrième gouvernement Berlusconi. B. Capproti est l'entrepreneur de référence des supermarchés Esselunga. Ces deux ouvrages appartiennent à une littérature pseudoscientifique qui reprend les lieux communs utilisés par les conservateurs au début $d u x x^{e}$ siècle
L 'interdépendance entre l'action politique municipale et la coopération a suscité récemment un regain d'intérêt de la part des historiens. L'étude de ce thème nécessite de mener une enquête sur un terrain habituellement perçu comme épineux: celui du mariage entre les affaires et la politique, ce qui explique que le débat historiographique soit tronqué par les jugements de valeur ${ }^{(1)}$.

Résumons en deux remarques les limites du récent débat historiographique dans ses différentes approches. Tout d'abord, la relation entre la coopération et la politique a toujours été traitée de manière restreinte, sans jamais élargir l'horizon à la relation entre l'entreprise et la politique. En Italie, mais pas seulement, l'entreprise opère dans un contexte - institutionnel, commercial, industriel, etc. - fortement conditionné par la politique, et c'est dans cet environnement qu'il faut rechercher les raisons de certains choix des entreprises. Ensuite, certaines décisions politiques sont étroitement influencées par le système productif. Tout cela, naturellement, peut 
ou durant la période fasciste: les coopératives vivotent au détriment de l'Etat et des contribuables, trompent les sociétaires au bénéfice des dirigeants, représentent un manifeste conflit d'intérêts, ne suivent que des buts clientélistes et pervertissent le marché libre. Une étude sérieuse a été publiée par Matia Granata en 2005. L'auteur, spécialiste de I'histoire de la coopération et collaborateur de Legacoop Lombardia, organisation régionale du mouvement coopérati de tradition socialiste, s'y efforce d'articuler une théorie de "I'agir coopératif " qui rende compte de la dialectique entre le système économique et le système politique. Il s'agit d'une contribution importante, représentant selon nous un point de départ et non un aboutissement pour les études historiques de ce genre.

(2) "Conception du monde " (NDLR). constituer soit un point fort, car cela signifie que l'Etat est très proche des exigences des entrepreneurs, soit une faiblesse, car cela évoque le spectre de la corruption et du clientélisme. Enfin, pour toute étude du lien entre la coopération et les partis, il convient de prendre en compte non seulement la relation entre l'entreprise et la politique, mais aussi la nature propre de l'entreprise coopérative. Cette dimension semble avoir curieusement échappé au débat historiographique.

Plutôt que de penser à ce lien comme à une sorte d' "échange ", il serait plus juste de parler d'une Weltanschaunng ${ }^{(2)}$ commune aux deux sujets, dans la mesure où ils partagent un univers éthique analogue et un objectif commun: le rejet d'une relation basée sur le clientélisme. En fait, le mouvement coopératif comme les forces politiques progressistes qui l'ont soutenu - marxistes, catholiques sociaux, républicains, libéraux démocratiques - ont toujours poursuivi l'objectif d'un changement de société. Celui-ci, à long terme, est le véritable but de la coopération, qui se propose comme élément de moralisation du marché, comme véhicule de modernisation économique et comme instrument de redistribution sociale (Zamagni, Felice, 2006, p. 223-231; Zamagni, 2008; Jossa, 2008). Ces caractéristiques sont absentes de l'entreprise classique, qui, même lorsqu'elle est animée par une logique éthique - nommée corporate social responsability (CSR) ou responsabilité sociale de l'entreprise (RSE) -, ne manifeste pas l'exigence de transformer la société.

Pour résumer, le débat historiographique sur la relation entre le mouvement coopératif et la politique devrait être actualisé à partir de ces deux considérations: englobée dans le dialogue entre l'entreprise et la politique, cette relation n'est pas exclusive, et elle se distingue par une finalité téléologique, celle de la métamorphose sociale et économique, qui est absente des préoccupations des entreprises privées.

Dans cet article, nous voulons démontrer que les logiques qui ont animé le mouvement coopératif en Emilie-Romagne entre 1945 et les années 70 n'étaient pas exclusivement économiques et étaient significativement différentes de la stratégie mise en place par l'entrepreneuriat traditionnel. Après avoir expliqué le rôle de l'entreprise coopérative sur l'activité de la reconstruction de l'après-guerre, nous évoquerons la collaboration entre les coopératives et les organismes locaux au cours des décennies 50 et 60 pour élaborer un plan de développement économique du territoire de l'Emilie-Romagne. Nous analyserons en particulier les quatre secteurs économiques où est présente la coopération: l'agriculture, la construction, la consommation et le crédit.

\section{La coopération et la reconstruction de l'après-guerre: des idées aux réalisations pratiques}

Voyons quelle fut l'évolution du mouvement coopératif dans le contexte des politiques municipales de l'Emilie-Romagne entre la période de la 


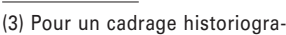
phique du mouvement coopératif, voir: Sapelli (1981); Zangheri et alii (1987); Fornasari, Zamagni (1997).

(4) Le Parti d'action (Partito d'azione), fondé en juillet 1942, fut un parti d'intellectuels influents. Ils avaient repris le nom du parti fondé en 1853 par Giuseppe Mazzini, disparu en 1867. Radical, républicain et socialiste modéré, le Parti d'action s'est dissous en 1947 (NDT).
Résistance et les années $70^{(3)}$. Il s'agit d'une relation qui se renoua au cours de la lutte de libération, après deux décennies fascistes d'humiliation et de mortification de l'esprit démocratique coopératif (Degl'Innocenti, 1995; Menzani, 2009a, 2009b), relation qu'il convient de distinguer en deux périodes. La première couvre la phase entre la fin de la guerre et l'après-guerre, à peu près de 1943 à 1950, et la seconde celle des années 50 et 60. Dans un premier temps, le lien entre la coopération et les administrations locales était si étroit qu'il fut qualifié d'« organique "; dans un second temps, ce lien a pris la forme d'une intégration mineure, suite à la participation d'autres organisations démocratiques comme - pour rester dans le milieu des entreprises - les chambres industrielles, commerciales et professionnelles.

\section{La coopération : un acteur parmi d'autres de la résistance antifasciste}

Au cours des débats initiés durant la Résistance sur l'organisation de l'Italie démocratique, la coopération s'est vu attribuer l'un des rôles principaux pour la construction morale et matérielle du pays. En fait, les trois grandes cultures politiques qui incarnaient la lutte antifasciste - les cultures libérale, socialiste et chrétienne - partageaient plus ou moins étroitement les idéaux du mouvement coopératif. L'entreprise autogérée fut reconnue par différentes organisations politiques comme l'une des formes organisationnelles à promouvoir après la guerre: les communistes, les socialistes, les sociaux-démocrates, les républicains, les catholiques et même les membres du Parti d'action ${ }^{(4)}$ et les libéraux ont confié aux coopératives un rôle moteur de gestion démocratique des ressources et du travail pour la phase postérieure à la Libération. Naturellement, chaque groupe avait une idée propre de ce qu'aurait dû être une coopérative et lui assignait un poids spécifique variable dans l'économie italienne libérée. $\mathrm{Si}$, pour les communistes et les socialistes, la coopération devait être le mode de production par excellence, en contraste avec l'initiative privée, pour les autres cultures politiques, en revanche, la coopération représentait plus simplement une opportunité au sein de la libre entreprise appelée à collaborer à la reconstruction.

Dans le feu de la Libération, ces différences interprétatives étaient mises sous l'éteignoir, surtout après la déclaration du secrétaire du Parti communiste italien Palmiro Togliatti, approuvée par les autres forces antifascistes, selon laquelle toute discussion sur l'aménagement de l'Italie devait être renvoyée après la capitulation des nazis-fascistes. Dans tous les cas, la propagande antifasciste a commencé à s'imprégner de coopération, comprise au sens générique comme le modèle économique et social à adopter une fois la guerre finie. De fait, après la Libération, dans différentes régions italiennes, mais en particulier en Emilie-Romagne, une véritable ferveur associationniste s'est diffusée, grâce à laquelle dès le printemps 1945 des centaines de nouvelles coopératives ont été constituées, alors que la croissance des entreprises traditionnelles était nettement plus modérée. 
La capacité de la coopération à faire face à la situation d'urgence alimentaire et de sous-emploi et sa crédibilité ont permis de concrétiser des expérimentations variées. En quelques mois, de nouvelles coopératives de manouvriers (braccianti), de maçons, de charretiers, d'artisans et de consommateurs ont vu le jour. Cette renaissance coopérative avait renoué avec la tradition préfasciste, même si en Emilie-Romagne, durant la phase initiale d'unité antifasciste, elle avait surtout été menée par les socialistes et les communistes, le plus souvent en collaboration avec les catholiques et les républicains (Menzani, 2007a).

Ce "miracle coopératif ", comme l'ont défini certains observateurs de l'époque, possédait les caractéristiques et les modalités relevant d'une Weltanschauung commune entre entreprises coopératives et forces politiques progressistes. Hormis l'enthousiasme fondamental des travailleurs, les nouvelles coopératives se sont également formées sur la base d'un input organisationnel important venant parfois d'autres milieux. Dans la majeure partie des cas, ce stimulus provenait de l'un des représentants des Comités de libération nationale (CLN), des partis traditionnels et des premiers gouvernements municipaux; ceux-ci étaient à même de remarquer si des travailleurs d'une même localité s'étaient réunis en une coopérative "de fait » ou si, dans un village particulier, une coopérative de consommation ou d'entraide agricole pouvait être utile. Ils s'appuyaient sur les principes et sur les motivations morales des travailleurs, les incitaient à construire juridiquement une entraide autogérée et, dans quelques cas, l'un d'eux en devenait président, directeur ou conseiller.

\section{Le rôle des autorités locales et de la société civile dans la promotion de la coopération}

Cette série d'initiatives réalisées à large échelle constitue donc la renaissance de la coopération en Emilie-Romagne durant le second après-guerre. A cheval entre les années 40 et les années 50, il y eut un premier moment décisif de sélection durant lequel les différents organismes provinciaux du mouvement, comme les fédérations et les consortiums, ont travaillé pour donner une structure plus rationnelle à cet ensemble d'initiatives disparates. Ainsi est né ce tissu coopératif, d'orientation Legacoop, qui dans les secteurs de l'agriculture, de la production et de la consommation a représenté un interlocuteur de premier ordre pour les nouvelles administrations publiques (Menzani, 2007a).

Dans l'urgence de la reconstruction qui a précédé le miracle économique, le mouvement coopératif a représenté un point de rencontre entre exigences sociales et économiques, puisqu'il a offert des opportunités aux chômeurs tout en favorisant le retour à la normalité, notamment par un dialogue constant avec les services publics locaux. Différentes recherches, fondées sur un solide corpus de sources inédites, ont montré que dans les années du second après-guerre la coopération incarnait un esprit fortement social, pas uniquement entrepreneurial, et qu'en ce sens elle a contribué de manière évidente à la reconstruction, comme l'illustre 
ce témoignage qui se réfère au second après-guerre: "Pour [...] les travaux absolument nécessaires et urgents le Génie civil, l'Electricité padane, la Préfecture et je ne sais qui d'autre [... devaient] intervenir. Mais tu peux attendre longtemps si tu attends ces gens. [...] C'est alors que la coopérative de maçons, qui n'a pas les moyens, mais a beaucoup de sociétaires avec une grande envie et un grand besoin de travailler, se propose. Et rapidement on franchira les ponts, bien que provisoires; les enfants pourront fréquenter l'école et le jardin d'enfants. Les sociétaires attendront [d'être payés]. C'est la coopérative des menuisiers qui s'occupe de réparer les portes, les fenêtres, les bancs, et qui patientera elle aussi pour l'encaissement. [...] Ce n'est pas un [...] problème que d'apporter [...] des galets pour les routes. Il y a la ligue des charretiers: "Ils nous payeront quand ils pourront, de toute façon les chevaux mangent aussi s'ils restent à l'écurie, les galets de la rivière ne coûtent rien; il s'agit seulement de notre fatigue." La coopérative de consommation faisait crédit. [...] Ensemble on se disait: "En fin de compte, la commune c'est nous, nous sommes toute une famille." [...] Il y a une carcasse d'autocar qui appartenait à l'armée: la coopérative des transporteurs la répare et lui fixe des bancs en bois. L'immatriculation et l'expertise auront lieu; en attendant l'autocar va et vient de Bologne deux fois par jour " (Argentesi, 1980, p. 289).

Dans une grande partie de la région, la relation entre la coopération et les administrations fut très étroite et orientée vers la satisfaction des besoins primaires, de la reconstruction des bâtiments aux politiques de détente des prix des biens de consommation, à la résorption du chômage et à différentes formes d'approvisionnement et d'assistance. Durant ces années, l'incertitude de la situation politique a très souvent gelé les investissements de l'industrie privée, ce qui permit à la coopération de gagner une extrême popularité. Le lien vertueux entre les administrations locales, le mouvement coopératif et la société civile avait alimenté de grands espoirs auprès des coopérateurs, qui ont souvent rêvé de pouvoir élaborer une nouvelle architecture économique.

\section{"Occasion manquée "}

L'hypothèse d'une solution transitoire possible, sans autre précision, d'intégration entre l'économie capitaliste et les principes marxistes avait permis d'envisager sérieusement un rôle de premier plan pour les coopératives. La Legacoop caressait l'idée d'un nouveau système productif fondé sur les entreprises autogérées, à partir d'une exclusivité pour les travaux publics, d'une réforme agraire fondée sur la location collective, et de nombreuses réductions fiscales pour permettre l'accès au crédit afin de rééquilibrer le rapport de force entre entreprises coopératives et les autres entreprises privées, dans la mesure où celles-ci n'accomplissaient pas une fonction sociale analogue. La défaite électorale des partis de gauche, au bénéfice de la Démocratie chrétienne, a sonné le glas des espoirs et des projets nourris par le Parti communiste et le Parti socialiste. Dans ces circonstances, les dirigeants de la Legacoop ont qualifié d'« occasion 
perdue " ce repli défensif du mouvement (Cerreti, 1959; Briganti, 1978). Presque simultanément, le mouvement a perdu son unité en EmilieRomagne. Lorganisation centrale des coopératives catholiques, la Confédération des coopératives italiennes (Confcooperative), s'est ramifiée en périphérie et la scission des composantes républicaines et social-démocrates de la Legacoop, survenue en 1951, a abouti à la création de l'Alliance générale des coopératives italiennes (AGCI). Suite à ces transformations, les années 50 et 60 seront caractérisées par une relation entièrement nouvelle entre le mouvement coopératif - avec ses différents orientations idéologiques - et les institutions politiques municipales de l'Emilie-Romagne.

\section{L'interlocuteur « démocratique »}

Sans entrer dans le détail, les gouvernements municipaux de l'EmilieRomagne des années 50 et 60 sont dominés par les forces politiques de gauche - c'est-à-dire des partis communistes et socialistes - ou de centregauche laïque - avec l'apport des sociaux-démocrates et des républicains et plus rarement par la présence de démocrates-chrétiens (Ballini, Ridolfi, 2002). La plupart du temps, ces exécutifs avaient le même point de vue que la principale composante du mouvement coopératif de l'EmilieRomagne, celle qui s'identifiait à la Legacoop et en partageait les valeurs. Le Parti communiste, en particulier, exerça une forte influence politique sur la coopération. En Emilie-Romagne, ce parti obtenait des résultats électoraux très nettement supérieurs à ceux de la moyenne nationale, parce qu'il était perçu d'une part comme le principal interprète de la lutte de libération, d'autre part comme l'héritier naturel de la tradition marxiste préfasciste, aux dépens surtout d'un Parti socialiste qui durant les années de clandestinité avait perdu beaucoup de terrain (Ferretti, 1982). Fort d'une excellente organisation, il avait pu passer rapidement du groupuscule extrémiste au grand parti de masse.

La coopération visait à long terme l'objectif, comme on l'a déjà mentionné, d'une transformation radicale de la société et du système économique. Sur cet objectif se sont greffées des exigences de redistribution sociale, de développement économique et de modernisation du territoire. Malgré des directions différentes, les exécutifs municipaux et le mouvement coopératif - auxquels on pourrait ajouter les forces syndicales de la Confédération générale italienne du travail (Confederazione generale italiana del lavoro, CGIL) - œuvraient pour un objectif commun. Tandis que les administrations accomplissaient une fonction publique, au service de la communauté et de la société civile, les coopératives avaient une tâche avant tout entrepreneuriale, partagée entre l'intérêt des sociétaires, qui restait la finalité principale, et un effet social plus large, lié aux exigences éthiques de la coopération.

La question des synergies entre l'action publique et la coopération au cours des décennies 50 et 60 mériterait que lui soit consacrée une 
(5) II s'agissait de coopératives de manouvriers qui prenaient en location un domaine agricole pour l'exploiter ensemble.

(6) Sur cette question, voir notamment: Ravaioli, Casadio, 1977 Landi, 1998; Muzzioli, Rinaldi, 1999; Rebeschini, 2005; Menzani, 2007b).

(7) Archives Progeo, section historique, enveloppe IV (divers), transcription du discours de F. Bassi en visite aux coopératives de Masone (Reggio Emilia), document daté du 25 septembre 1961 recherche approfondie. Nous nous bornerons ici à en présenter les tendances principales et à esquisser quelques interprétations, de manière à ouvrir des pistes pour des études historiques ultérieures. La coopération s'est intéressée principalement à quatre secteurs économiques: le secteur agricole (et agroalimentaire) et ceux de la construction, de la consommation, et du crédit.

\section{La coopération, moteur de la modernisation agricole}

Dans les campagnes, les coopératives ont été les vecteurs de la transition et de la modernisation agricole. En fait, le modèle des «locations collectives ${ }^{(5)}$ " a été progressivement abandonné au profit d'un réseau de plus en plus dense de coopératives entre agriculteurs pour la commercialisation ou la transformation de leurs produits. Des milliers de petits propriétaires, de métayers ou d'agriculteurs en location ont réussi à parler d'une seule voix sur le marché et à défendre leurs investissements et leurs revenus. En ce sens, la coopérative agricole a contribué à conduire une transformation rurale radicale qui, sans cette intervention active, aurait risqué de provoquer des coûts sociaux énormes ${ }^{(6)}$. De fait, certaines zones de campagne de la plaine du Pô ou des Apennins subissaient une diminution et le vieillissement de la population, tandis que les zones à vocation industrielle risquaient d'être littéralement submergées par une urbanisation chaotique. La capacité de la coopération agricole à maîtriser ce processus de modernisation et à le répartir dans le temps - aussi sous forme de « résistance »- a permis aux administrations publiques de se préparer à l'« exode rural » et de mettre au point de nouveaux plans d'urbanisme, de nouveaux services et de nouvelles politiques sociales en prévision de ces transformations. "Aujourd'hui encore plus que par le passé, les coopératives agricoles de manouvriers et les paysans ont le devoir de sauvegarder le travail des sociétaires qui veulent continuer à rester en contact avec le monde de la campagne, déclare ainsi un dirigeant émilien de la Legacoop en 1961. [...] Pourquoi faut-il émigrer à Reggio, Modène ou Bologne ou, encore pire, à Milan, à Turin ou même à l'étranger? Qui a dit qu'il faille le faire? Nous, coopérateurs agricoles, nous devons lutter pour rester dans nos campagnes et pour "coopératiser" tout ce qui reste encore en main des receveurs de rente, aux dépens de la péréquation sociale. Celui-ci est le grand mal qui continue d'affiger notre agriculture ${ }^{(7)}$."

\section{L'habitat coopératif}

Cette situation a engendré l'établissement d'une relation positive entre les administrations locales et les coopératives de construction et d'habitation, qui constituent le second objet de notre étude. Une fois la reconstruction terminée, lorsque le miracle économique a commencé à dissiper les difficultés de l'après-guerre, le territoire régional a vu poindre de nouvelles exigences. Des infrastructures publiques devaient être construites, comme des routes, des voies ferrées, des canalisations de bonification, des réseaux de distribution d'eau, des centrales électriques, des écoles, 
(8) L'United Nations Relief and Rehabilitation Administration (UNRRA) était une organisation des Nations unies instituée en 1943 pour aider les pays gravement endommagés par la Seconde Guerre mondiale. Son œuvre fut ensuite élargie aux pays vaincus. En Italie, suite aux accords de Rome du 8 mars 1945 et du 19 janvier 1946, I'UNRRA fut autorisée à instituer une mission qui exerçait ses fonctions par l'intermédiaire de commissions provinciales présidées par le préfet et par les comités communaux, lesquels étaient chargés de distribuer les aides. L'UNRRA fut abolie le 3 décembre 1947 (Gustafson, 1966).

(9) II s'agissait d'un plan d'intervention publique pour la réalisation d'habitations à loyers modérés sur tout le territoire italien avec des fonds gérés par une organisation ad hoc auprès de I'Institut national des assurances (INA).

(10) Sur cette question, voir notamment: Sapelli, Zan (1991); Canova (2001); Pedrocco (2001); Menzani (2003); Furlan, Ottan (2006); Sapelli (2008). des hôpitaux et des casernes. Le besoin d'une sérieuse planification d'habitations se faisait aussi sentir, afin de résoudre le «problème de l'habitation " et de réagir à une croissante carence de logements dans les zones touchées par l'urbanisation. Dans les périphéries des villes, de même quau sein des communes des hinterlands, il fallait penser à construire des quartiers résidentiels et des zones industrielles pour accueillir l'exode rural (Pierini, 2001; Parisini, 2003, 2006).

Dans ce but, les administrations locales, en accord avec le gouvernement central, avaient commencé à appliquer une série de directives faisant suite aux anciens plans UNRRA qui avaient caractérisé la reconstruction de l'après-guerre ${ }^{(8)}$. Une fois ces projets terminés, le plan INA-Casa ${ }^{(9)}$ a pris le relais - passé en 1963 à l'Institut de gestion des habitations des travailleurs (Istituto gestione case lavoratori, Gescal) -, suivi des plans de l'habitation économique populaire (Piani di edilizia economica popolare, PEEP). Les instituts autonomes des habitations populaires (istituti autonomi case popolari, IACP), nés au début du xxe siècle comme institutions morales, étaient aussi de la partie. En pratique, au niveau local, une série d'organismes publics ou parapublics se préoccupaient de planifier une croissance ordonnée pour répondre aux nouveaux besoins de logement. Le mouvement coopératif fut pour eux un interlocuteur de grande importance, par l'intermédiaire des coopératives de construction, qui regroupaient des maçons, ou par l'intermédiaire des coopératives d'habitations. Il ne s'agissait pas seulement d'une simple relation économique ou commerciale entre une demande et une offre, entre celui qui avait besoin d'une habitation et celui qui se proposait de la construire. En Emilie-Romagne, Legacoop opta pour une approche éthique. Elle refusa par exemple d'adopter la pratique de construire à son propre compte pour vendre ensuite au prix du marché - comme le faisaient les entreprises privées du bâtiment - et choisit de travailler uniquement pour la construction populaire ou civile, sur commande de l'IACP ou d'autres sujets publics, avec des marges minimes de profit: "La tendance au travail à caractère spéculatif, [c'est-à-dire] la construction d'immeubles à vendre sur le marché privé, même si cela peut parfois donner des résultats positifs, comporte des immobilisations importantes et risquées, et mène ainsi la coopérative du champ naturel de la sécurité et de la prudence à celui de la spéculation privée qui, entre autres, n'est certainement pas celui dans lequel les coopératives doivent opérer si elles sont bien dirigées et conscientes de la fonction quielles doivent accomplir ", expliquait ainsi la fédération de Bologne de la Ligue nationale des coopératives $(1954$, p. 53$)$.

Tout cela a produit, dans les années 50 et 60 , une véritable détente des prix dans le secteur du bâtiment et a permis en conséquence aux classes moyennes inférieures d'accéder à coûts modestes au bien primaire de l'habitation ${ }^{(10)}$.

Il s'agit peut-être de l'exemple le plus éclatant et le plus vertueux de relations entre les gouvernements municipaux et le mouvement coopératif. La société civile de l'Emilie-Romagne a bénéficié des convergences entre 
(11) Sur ce point, voir notamment : Battilani (1999) ; Menzan (2009c) ; Casali (2000) ; Zamagni et alii (2004).
(12) Archives Coop Adriatica, siège de Ravenne, matériaux historiques, Cooperativa di produzione e consumo della provincia di Ravenna, procès-verbal du CA 5 octobre 1971. la politique des travaux publics ou des habitations populaires et celle des entreprises autogérées engagées dans leur réalisation, et grâce à cette confluence elle s'est dotée, à coûts plus modérés que dans d'autres régions, d'infrastructures fonctionnelles et d'un bon nombre de nouvelles habitations (Bortolotti, 1978).

\section{Modernisation du commerce}

La coopération de consommation et les administrations locales ont surtout interagi dans l'immédiat après-guerre, en exerçant une précieuse fonction de détente des prix. Puis, dans les années 50 et 60, la coopération de consommation s'est engagée dans un processus graduel de rationalisation, par la fusion de petits magasins coopératifs limitrophes, la création de consortiums d'achat et, surtout, l'introduction d'un nouveau type de points de vente comme les premiers supermarchés self-service. En un certain sens, la coopération de l'Emilie-Romagne a incarné l'esprit modernisateur du commerce et contribué à son développement ${ }^{(11)}$. Le préjugé selon lequel la distribution commerciale serait un élément secondaire de la croissance économique est aujourd'hui définitivement abandonné, tant le sous-développement du système distributif national - au sein duquel les formes traditionnelles du commerce ont survécu pendant longtemps - est considéré comme l'une des raisons cruciales de l'écart entre les Etats-Unis et l'Italie en ce qui concerne l'augmentation de la productivité du travail. En l'espèce, le travail en synergie avec les services publics locaux, dispensateurs de licences, de permis et d'autorisations, a transformé progressivement le réseau de vente. Dans l'ensemble des provinces de l'Emilie-Romagne, cela a conduit à la suppression graduelle des petits magasins coopératifs pour faire place aux premiers supermarchés et amorcé un renforcement de la grande distribution moderne: "Le président [de la Coop Adriatica] s'attarde longuement sur les nouvelles structures dont la coopération de consommation de l'Emilie-Romagne doit se doter. Les entreprises devraient être de niveau national, autogérées par les sociétaires, avec un réseau de vente valide et moderne. En synthèse il faudrait arriver à: 1) une restructuration de l'entreprise, 2) une restructuration du réseau de vente, 3) un nouvel aménagement des services en amont. [...] Mais avant, il est nécessaire de connaître la situation économique de chaque coopérative et de regarder le programme organique pour éliminer les petits magasins coopératifs traditionnels, [...] en accord avec les administrations locales et les autres sujets concernés. Il n'est pas possible de réformer le réseau de vente sans un plan commun ${ }^{(12)}$."

\section{Développement du crédit coopératif}

En dernier lieu, le crédit coopératif, contrairement aux secteurs déjà évoqués, se distinguait par une nette prépondérance de l'influence catholique. Les nombreuses caisses rurales et d'artisans, devenues des banques de crédit coopératif, étaient sans aucun doute la pointe la 
plus avancée du mouvement coopératif appartenant à Confcooperative et représentaient un élément important du système du crédit régional. Même si durant les années 40 et 50 plusieurs de ces organismes ont subi une forte crise, il y eut, durant la décennie suivante, une reprise généralisée sur laquelle l'historiographie a beaucoup insisté (Cova, Scidà, 1983; Fornasari, 1997; Cafaro, 2002). Même dans ce milieu, malgré certaines frictions de caractère politico-idéologique, d'importantes synergies entre ces caisses rurales et les administrations locales ont émergé, surtout pour le soutien de la petite entreprise.

Comme plusieurs auteurs l'ont souligné, les années du miracle économique ont connu une politique de stimulation de l'artisanat et de l'industrialisation diffuse, soutenue par les banques territoriales, notamment coopératives. Il s'agissait d'une collaboration très importante entre la petite entreprise, le système bancaire local et les administrations publiques. Elle aura contribué positivement au modèle de développement des districts industriels, des entreprises en réseau et des activités artisanales qui caractériseront la région et contribueront à un accroissement sensible de la qualité de vie.

\section{Conclusion}

Par ce bref exposé, nous avons voulu montrer les liens qui dérivent de la collaboration entre les institutions locales et les entreprises, en particulier entre les administrations publiques et le mouvement coopératif. Même dans une perspective historique, il serait réducteur de définir cette relation en termes d'échanges. De plus, il apparaît avec évidence que la liaison politico-institutionnelle a été cultivée sous une forme vertueuse qui appartient à l'ensemble des dispositions et des stratégies génériquement appelées « lien avec le territoire ».

La tendance à l'établissement du dialogue entre l'Etat et la société civile se décline à l'échelle micro-spatiale, même par l'intermédiaire de relations de courtoisie entre services publics locaux, partis politiques et entreprises: un conseiller municipal est invité à l'inauguration d'un nouvel établissement; les représentants de la coopération assistent à la discussion du bilan communal; les politiciens et les présidents des diverses organisations de représentation siègent côte à côte lors des événements mondains. Ces relations sont généralement perçues par le sens commun comme partiellement négatives, liées à la corruption, au clientélisme et à la spéculation, mais en réalité - hormis quelques dérives frauduleuses - elles sont vitales pour rapprocher et recomposer les fractures entre l'Etat et la société, entre la sphère sociale et le marché, entre les classes dirigeantes et les travailleurs. La " relation avec le territoire " est une manière de croiser les synergies pour la résolution des besoins, la valorisation des ressources, l'exploitation des opportunités et le développement des circonscriptions. 
En résumé, nous pouvons affirmer que la collaboration entre le mouvement coopératif et les administrations municipales de l'EmilieRomagne du second après-guerre et du miracle économique a suivi deux phases distinctes: un temps de grande complémentarité entre les deux sujets politiques, résultant des besoins et des rêves de ce moment historique particulier, suivi par un temps de collaboration moins étroite, au cours duquel le mouvement coopératif n'était que l'un des différents interlocuteurs des administrations locales, tout en continuant à en partager les valeurs, une vision commune de la société et la même volonté de la changer positivement. L'entreprise coopérative fut ainsi un véhicule de modernisation qui, en syntonie avec les administrations locales, porta une société encore largement rurale vers de nouveaux standards de bien-être. 


\section{Bibliographie}

Argentesi D., 1980, Nelle case et per le strade di un borgo emiliano: ricordi di un militante comunista, Bologna, Feltrinelli.

Ballini P. L., Ridolfi M. (a cura di), 2002, Storia delle campagne elettorali in Italia, Milano, Bruno Mondadori.

Battilani P., 1999, La creazione di un moderno sistema di imprese: il ruolo dei Consorzi della cooperazione di consumo dell'Emilia-Romagna, Bologna, Il Mulino.

Bortolotti L., 1978, Storia della politica edilizia in Italia: proprietà, imprese edili e lavori pubblici dal primo dopoguerra ad oggi (1919-1970), Roma, Editori Riuniti.

Briganti W. (a cura di), 1978, Il movimento cooperativo in Italia, 1926-1962, Roma-Bologna, Editrice cooperativa-Ape.

Cafaro P., 2002, La solidarietà efficiente: storia e prospettive del credito cooperativo in Italia (1883-2000), Roma-Bari, Laterza.

Canova F., 2001, Cooperativa Muratori Reggiolo: 90 anni di vita e lavoro, 1907-1997, Reggiolo, Circolo Polaris, 1997.

Caprotti B., 2007, Falce e carrello: le mani sulla spesa degli italiani, Venezia, Marsilio,

Casali A., 2000, Per una storia di Coop Italia: Mario Cesari (1926-1968), Bologna, Il Mulino. Cerreti G., 1959, "L'occasione perduta ", Movimento cooperativo, luglio-ottobre.

Cova A., Scidà G. (a cura di), 1983, Cooperazione di credito e sviluppo sociale ed economico delle campagne in Emilia Romagna, Bologna, Federazione delle casse rurali ed artigiane dell'Emilia-Romagna.

Degl'Innocenti M., 1995, La società unificata: associazione, sindacato, partito sotto il fascismo, Manduria, Lacaita.

Federazione di Bologna della Lega Nazionale delle Cooperative, 1954, Due anni di attività dei cooperatori bolognesi, Bologna, Steb.

Feltri V., Brunetta R., 2007, Le coop rosse, il piu grande conflitto di interessi nell'Italia del dopoguerra, Free Foundation.

Ferretti V., 1982, Riformisti di Lenin: la cooperazione reggiana nel secondo dopoguerra, Reggio Emilia, Tecnostampa.

Fornasari M., 1997, Il credito cooperativo in Emilia Romagna: le strutture di secondo livello dalla ricostruzione alle soglie del duemila (1945-1996), storia e testimonianze, Bologna, Federazione delle banche di credito cooperativo dell'Emilia-Romagna.

Fornasari M., Zamagni V., 1997, Ilmovimento cooperativo in Italia, un profilo storico-economico (1854-1992), Firenze, Vallecchi.

Furlan P., Ottani V., 2006, Noi della Selciatori: 70 anni di Coop Costruzioni, Bologna, Clueb. Granata M., 2005, Impresa cooperativa e politica: la duplice natura del conflitto, Milano, Bruno Mondadori.

Gustafson M. O., 1966, Congress and foreign aid: The first phase, Unrra, 1943-1947, Ann Arbor, University of Nebraska.

Jossa B., 2008, L'impresa democratica, un sistema di imprese cooperative come nuovo modo di produzione, Roma, Carocci.

Landi F., 1998, Storia di una cooperativa: braccianti imprenditori del comprensorio di Cervia, 1904-1970, Ravenna, Longo.

Menzani T., 2003, La cooperazione edile nel Lughese e nel Faentino dalla Liberazione alla RES Coop, Ravenna, Longo.

Menzani T., 2007a, La cooperazione in Emilia-Romagna. Dalla Resistenza alla svolta degli anni settanta, Bologna, Il Mulino.

Menzani T., 2007b, «"Lanticamera del kolkhoz sovieticoe": i collettivi agricoli nell'EmiliaRomagna del secondo dopoguerra ", Rivista di storia dell'agricoltura ", n 1, p. 131-152.

Menzani T., 2009a, Il movimento cooperativo tra le due guerre, il caso italiano nel contesto europeo, Roma, Carocci. 
Menzani T., 2009 b, « La cooperazione nell’Europa degli anni Venti e Trenta: un quadro comparativo ", La rivista della cooperazione, $\mathrm{n}^{\circ} 2$, p. 5-12.

Menzani T., 2009c, "Alla ricerca della convenienza, la cooperazione di consumo ravennate da una logica di calmierazione ad una di efficienza (1943-1978) ", in Andrea Baravelli (a cura di), Un secolo di cooperazione di consumo di Ravenna (1861-1980), Ravenna, Longo, p. 203-273.

Muzzioli G., Rinaldi A., 1999, Un secolo di cooperazione: la Cpl Concordia dal 1890 al 1999, Bologna, Il Mulino.

Parisini R. (a cura di), 2006, Politiche urbane e ricostruzione in Emilia-Romagna, Bologna, Bononia university press.

Parisini R. (a cura di), 2003, I piani della città: trasformazione urbana, identità politiche e sociali tra fascismo, guerra e ricostruzione in Emilia-Romagna, Bologna, Compositori.

Pedrocco G., 2001, 1901, 2001: Cmc cent'anni, Ravenna, Cmc.

Pierini R., 2001, La città distante: piani e progetti di edilizia residenziale pubblica, Pisa, Ets. Ravaioli A., Casadio C., 1977, Storia della Cooperativa agricola braccianti di Mezzano: settant'anni di lotteper lo sviluppo delle campagne, Imola, Galeati.
Rebeschini M., 2005, Amore per la terra: icinquant'anni della Cooperativa agricola braccianti Giulio Bellini, Bologna, Moderna.

Sapelli G. (a cura di), 1981, Il movimento cooperativo in Italia, storia e problemi, Torino, Einaudi.

Sapelli G., 2008, L'impresa per la giustizia sociale: storia della Cmb Cooperativa muratori e braccianti di Carpi, Milano, Bruno Mondadori.

Sapelli G., Zan S., 1991, Costruire l'impresa: la Cooperativa muratorie cementisti di Ravenna dal 1945 al 1972, Bologna, Il Mulino.

Zamagni S., Zamagni V., 2008, La cooperazione, tra mercato e democrazia economica, Bologna, Il Mulino.

Zamagni V., Battilani P., Casali A., 2004, La cooperazione di consumo in Italia, centocinquantanni della Coop consumatori: dal primo spaccio a leader della moderna distribuzione, Bologna, Il Mulino.

Zamagni V., Felice E., 2006, Oltre il secolo, le trasformazioni del sistema cooperativo Legacoop alla fine del secondo millennio, Bologna, Il Mulino.

Zangheri R., Galasso G., Castronovo V., 1987, Storia del movimento cooperativo in Italia (1886-1986), Torino, Einaudi. 